\title{
Quantitative analysis of the influence on the detection level of the data assigned to channels in optical thermographic RGB projected images
}

\author{
by P. Venegas*, R. Usamentiaga**, J. Perán***, I. Sáez de Ocáriz* and L. Vega* \\ * Centro de Tecnologías Aeronáuticas, Parque Tecnológico de Álava, Spain, pablo.venegas@ctaero.com \\ ** Universidad de Oviedo, Asturias, Spain, rusamentiaga@uniovi.es \\ *** Universidad Nacional de Educación a distancia, Madrid, Spain, jperan@ind.uned.es
}

\begin{abstract}
Optical stimulated IRT is rapidly becoming an important NDT tool for quality control thanks to its features of contactless remote inspection and high data acquisition rate; among others. However, this velocity capacity sometimes becomes an important drawback because of the great amount of data generated in the inspections, which must be subsequently analyzed.

The RGB projection method is a data synthesis technique very useful in this situations. To contribute to develop an effective methodology for RGB projection application, this study deals with the quantitative analysis of the influence of the content of each RGB channels on the final detection level.
\end{abstract}

\section{Motivation}

Optical stimulated infrared thermography is rapidly becoming an important NDT tool for materials quality control thanks to its features of contactless remote inspection and high rate of data acquisition; among others. This allows the application of Optical IRT in a wide range of conditions and types of material. However, this quick capacity of application provided by the technology sometimes becomes an important drawback because of the great amount of data generated in the inspection, which must be subsequently analyzed to finally determine the state of a material.

Typically, after a thermographic inspection the entire recorded sequence of images, which sometimes can reach the order of thousands of images, is reviewed. This procedure is applied because the instant that a defect reveals its presence in a thermographic inspection depends on the depth at which it is located. This means that we can have information of interest at different instants of the recording and therefore it must be reviewed entirety. Additionally it means that we cannot have grouped visualization of all indications in their optimal detection, but a different image for each indication, which must be analyzed independently to proceed with the characterization. This makes the global time consumption in the inspection not affordable for all the NDT applications.

To reduce this time consumption in the analysis stage different data synthesis techniques, such as PCT, were introduced in the IRT NDT field providing a partial solution. More recently, the RGB projection method has also been formulated offering additional improvements. The RGB method constitute an effective methodology of collecting the information of optimal detections obtained under different inspection conditions into one single image, so the analysis time dependence is drastically reduced. This provides an important advance in the analysis stage of IRT inspections. However, the procedure for optimizing the RGB image is currently under development. Therefore, in order to contribute to the development of an effective methodology, this study is focused in the quantitative analysis of the influence of the content of each RGB channels on the final detection level for improving the final result of the projection.

\section{Thermographic inspections used in the analysis}

In this study several defects of different nature were selected to be thermally excited using an optical method for IRT inspection. Specifically, optical step heating thermography was the one selected among the different stimulation options. It was selected due to the possibility to control the injection of energy into the material during the entire inspection, which allows a precise control of the highest temperature level, preserving at the same time the capacity of non-contact inspection of the IRT technology.

In addition to the results of the IRT inspections presented in images, the numerical data of temperature field were further processed to improve the detection of indications that can identify the presence of anomalies. The postprocessing techniques include the application of various types of mathematical algorithms to data acquired in the inspections. With its application improved detection levels are achieved, making possible the location of defects which might not be detected in the initial results (raw). The post-processing techniques used in this study include the application of various types of mathematical algorithms such as:

- $\quad$ Fourier Transform

- Thermal Signal Reconstruction (TSR)

- Statistical moments

- Wavelets 
- Principal component decomposition

$\bullet \quad \ldots$

\section{Preliminary results}

The selected specimens containing defects were inspected by optical IRT and subsequently processed with various algorithms. Among the results obtained after this procedure some characteristic images were selected for candidates to be included in RGB channels. The criteria followed for selecting these images were based on different optimality conditions such as the individual and combined values of SNR and sharpness, among others. Some preliminary results are shown in Figures 1 and 2.

Finally, the RGB projection of the selected candidate images was carried out by means of the application different combination strategies. Afterwards the level of detection produced by determining the final SNR value was quantified and so evaluated the influence of the information included in each channel.
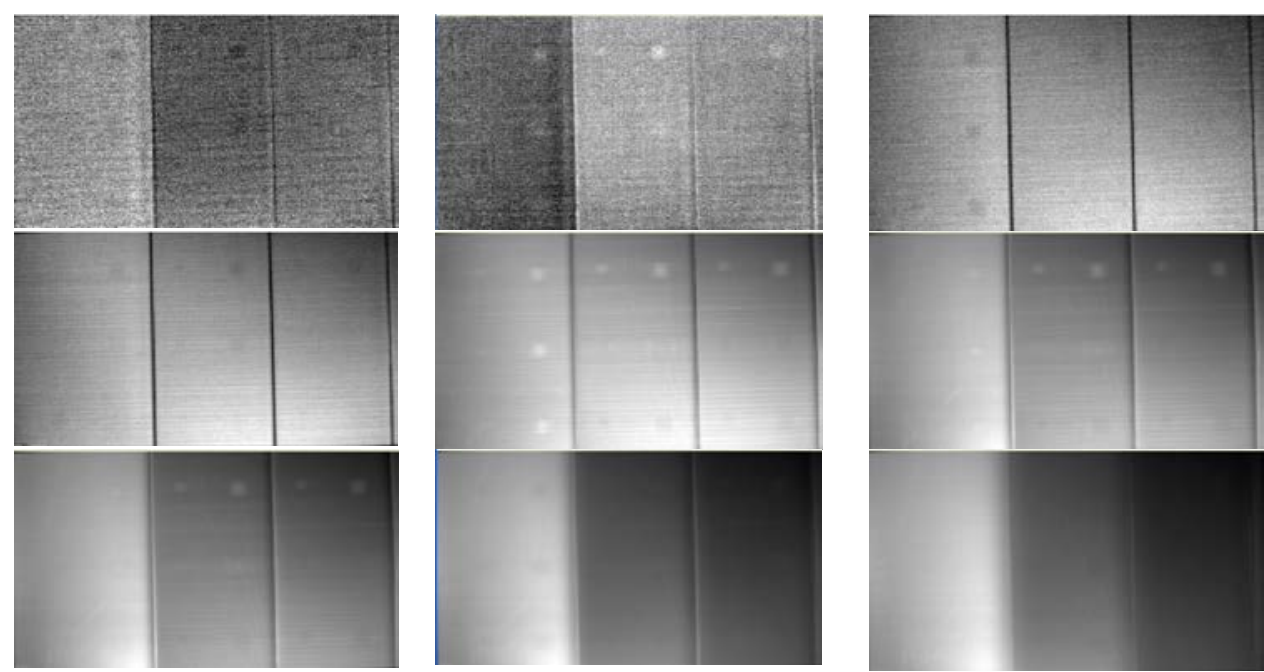

Fig. 1. Candidates for RGB channels under condition of relative extrema of SNR.
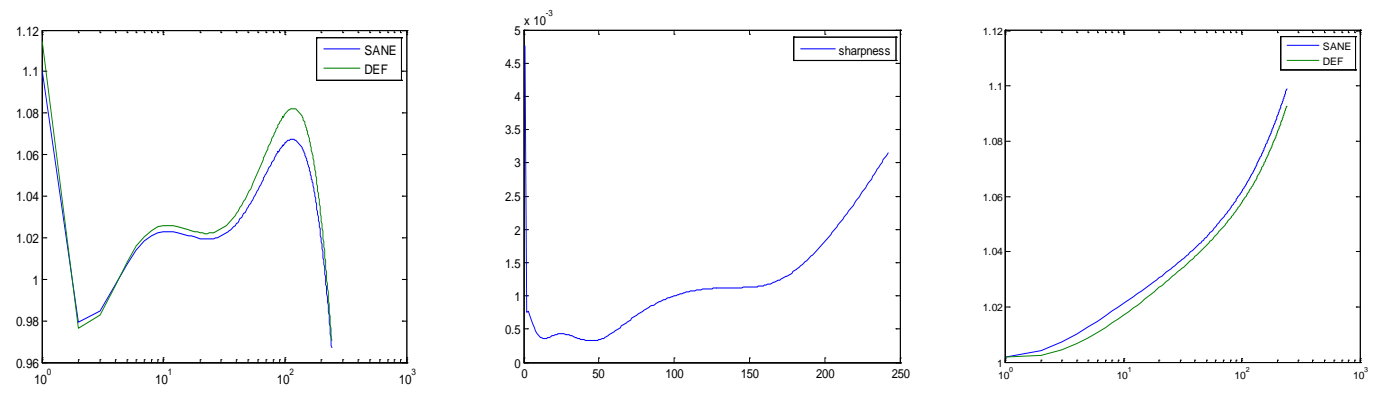

Fig. 2. Values of SNR and sharpness corresponding to thermal time history of a defect.

\section{REFERENCES}

[1] Shepard S. M., Lhota J. R., Rubadeux B. A., Wang D., and Ahmed T., "Reconstruction and enhancement of active thermographic image sequences," Optical Engineering, vol. 42, no. 5, pp. 1337-1342, 2003.

[2] Meola C. and Carlomagno G. M., "Recent advances in the use of infrared thermography," Measurement science and technology, vol. 15, no. 9, p. R27, 2004.

[3] Ibarra-Castanedo C., Gonzalez D., Klein M., Pilla M., Vallerand S., and Maldague X., "Infrared image processing and data analysis," Infrared physics \& technology, vol. 46, no. 1, pp. 75-83, 2004.

[4] Balageas D. L., "Defense and illustration of time-resolved pulsed thermography for NDE," Quantitative InfraRed Thermography Journal, vol. 9, no. 1, pp. 3-32, 2012.

[5] Roche J.M., Balageas D., "Detection and characterization of composite real life damage by the TSR polynomial coefficients RGB projection technique", 12 ${ }^{\text {th }}$ Quantitative Infrared Thermography Conference, 2014. 